\title{
DENTAL PATIENTS;
}

ROLE OF MUSIC DISTRACTION IN THE MANAGEMENT OF APPREHENSIVE DENTAL PATIENTS.

1. BDS, MSc, FCPS (Operative Dentistry)

Associate Professor \& Chairman Institute of Dentistry, LUMHS, Jamshoro.

2. BDS, Lecturer Jinnah Sindh Medical \& Dental College, Karachi.

3. BDS, MSc (Restorative Dentistry), Lecturer Restorative Dental Sciences,

Faculty of Dentistry

King Khalid University, Kingdom of Saudi Arabia.

4. BDS, Msc

Senoir Dental Surgeon

Civil Hospital, Karachi.

Correspondence Address:

Dr. Abdul Qadir Khan

Institute of Dentistry,

LUMHS, Jamshoro.

qadir.dall@lumhs.edu.pk

Article received on:

28/07/2018

Accepted for publication:

$15 / 12 / 2018$

Received after proof reading: 26/03/2019

\section{INTRODUCTION}

Dental anxiety has been noted to be a major and a very common problem in both children and adolescent. It is frequently seen as a fear that something bad is going to happen when going for the dental treatment and is also attached with the sense of losing control over the situation. ${ }^{1}$ Anxiety is a more general unfocused feeling of discomfort or uneasiness that is directly related to fear surrounding an unusual environment. ${ }^{2}$ Dental anxiety can be defined as a "patient's response to the stress that is specific to the dental situation". ${ }^{3}$

"Anxiety is more specifically characterized by subjective, consciously perceived feelings of danger, nervousness, and tension accompanied by autonomic nervous system arousal. Behaviors associated with anxiety include restlessness, trembling, fearful facial expressions, and shortness of breath, muscular tension, and fatigue". ${ }^{4}$ There are multifactorial aetiology of dental anxiety occurrence such as watching dental instruments, surrounding noise the two most anxiety arousing being the injection and the drill sound. ${ }^{5}$ Typically dental anxiety is accompanied by physiological and psychological responses such as increased heart rate, increase blood pressure, altered respiration rate, sweating, trembling, feelings of coming danger and tension. The dentist's attitude and manner and the dental environment also play a vital part in dental anxiety. ${ }^{6}$ Dentistry in modern era has struggled hard to provide an environment which is patient friendly. Anxiety related to dental procedure rank 4 among the common fears and $9^{\text {th }}$ among the intense fear that people face in their lives. ${ }^{7}$ The global findings estimated that more than $75 \%$ of the adults in United States experience some degree of mild to moderate anxiety and of them 5 to $10 \%$ are fearful regarding dental treatment hence they avoid any dental care needed $^{8,9}$ and these 5 to $10 \%$ of the people only seek dental care when they face severe dental problems such as severe pain and swelling leading that further lead to traumatic invasive procedures. $^{7,8,9}$ Many patients avoid visiting dentist just because of experiencing anxiety. 
Literature reports that many patients consider pain the primary source of anxiety consequently creating a hindrance in seeking dental care or it can be other way round that anxiety can lead to intense feeling of pain during locally administered anesthesia. ${ }^{10}$

With the progress in the dental field, different management ways have been introduced and now has been used in order to make the procedure less anxiety provoking and less painful for the patient. Sedatives and analgesia have always remained important with respect to the dental procedures since it allows dentist to treat the need and receive less distraction from the patient side and also allowing patient to experience less pain and anxiety. Apart from the above stated ways of treating anxiety and pain, there are other ways also that revolves around cognitive and behavioral aspect of the person consisting of relaxation techniques, deep breathing exercises, guided imagery and many others. Besides that, there is an important notion that has emerged from the past and has also received tremendous importance in present in different domains of medicine is music. ${ }^{5,6}$

Dental anxiety is manageable. For the dental practitioner, it is important to know that dental apprehension is a continuum ranging from mild to more severe form rather than a yes and no process. Milder forms of anxiety can be managed at initial levels with a treatment style that acknowledges such feelings and decreases them with appropriate management approaches, including coping strategies and pharmacological interventions. While anxiety in its more complex forms may need a team approach involving psychologists or psychiatrists. ${ }^{11}$ It would be a good approach if treating dental practitioners assess the dental anxiety and other related needs of their patients prior to dental procedures and then use appropriate.

Of the above mentioned management techniques, Music has been equally important in past as well as has gained due significance in present. "Music is defined as a complex of expressively organized sounds composed of few key elements: rhythm, melody, pitch and harmony". ${ }^{12}$ Music has its roots embedded in past. In ancient times, music has been used to treat certain medical conditions to restore the mind and body harmony. Pythagoras, who was a sixth-century Greek philosopher and also the founder of geometry and music therapy, believed that music had great impact on health of an individual; therefore, he often prescribed music and diet to reestablish and preserve the body and soul harmony. ${ }^{12}$ Likewise in recent years music has gained significant importance and music therapy is being used in various medical settings such as intensive care unit, surgery, psychiatry and cancer treatment for both adults and children. ${ }^{12}$

Music can actually calm patients, improve vital signs and enhance patient's overall levels of relaxation. ${ }^{13}$ Music act as an audio analgesic in dentistry and music can relieve pain and anxiety by moving conscious thought away from the symptoms. ${ }^{14}$ Studies have reported the usefulness of music in the preoperative settings where its major role is in alleviation of preoperative anxiety. ${ }^{12}$

Therefore; aim of this study isto investigate the association between use of music during the dental procedures (restorative or endodontics) along with the routine care and anxiety level of the participants.

\section{MATERIAL AND METHODS}

The study was undertaken as a hospital based randomized, controlled trial. The study was conducted in the Outpatient Departments of Operative Dentistry, at the Liaquat University Hospital, Jamshoro Hyderabad. The duration of the study was 1 year and data collection started from January 2014 to December 2014. The total sample size for this study was 200 participants. 100 participants in intervention (music) arm and 100 in the control (no music) arm. The sample size is calculated using WHO recommended sample size determination software for health studies and also by considering the result of previous international study as a reference..$^{10}$ The sampling technique used for this study was purposive sampling. Those participants were included 
in the study who met the eligibility criteria. Patients were selected if they met the eligibility criteria for the study. Patients were approached in the OPD where initial assessment was done and history was taken. Based on the eligibility of patient, informed consent was sought and then patient was included in the study in one of the two arm based on the lottery method. We included patients from 18 to 60 years of age of both genders who shown willingness to listen music during dental procedure (Dentist rating of patient scale 3-4 (medium) or 1-2 (high) and having no hearing impairment). We also checked that patient is apprehensive and should not have received any preoperative sedative or analgesic which can affect vital signs and can mask anxiety. We excluded the patients $>60$ years with any systemic medical disease. Uncooperative patients and patients low on dentist rating of patient scale 5-6 (low).

\section{Data Collection Procedure}

Patients presenting in the OPD of Operative Dentistry at Liaquat University Hospital Hyderabad, were selected for this study. History was taken and diagnosis was made. Eligibility was checked and informed consent was taken from the participants. Dentist rating scale was applied and score was checked. According to this scale; the levels of patient's treatability will be assessed as low (from 5 to 6 points), medium (3-4) and high (1-2) by the dentist by asking questions: how would you describe your tension during the dental treatment. ${ }^{15,16}$ Patients were equally divided in to the two groups: group A (intervention arm) and group B (control arm). In both groups the routine usual care was given. But group A being the intervention arm received music during dental procedure (restorative or endodontics). In group A anxiety was assessed using the dentist rating of patient's scale pre procedure. All patients were managed by single operator. Patients were given their preferred music based on their preference of particular song or singer along with comfortable set of headphones in order to divert patient's mind and to reduce anxiety. If during procedure any problem was faced by patient, music was stopped and then direct communication was made with the patient. Likewise after the procedure again the dentist rating of patient's scale was applied and patients' anxiety was assessed accordingly.

Group B were also assessed for anxiety using the Dentist rating of patient's scale pre and post procedure. Patients in this group didn't receive any music distraction. Dental procedure was carried out as per routine.

\section{STUDY VARIABLES}

Study variables for this study include:

\section{Independent Variable}

1. Use of music: Music was noted categorically as $1=$ yes for those who received music distraction (Group A) and $0=$ no for those who didn't receive music distraction (Group B). Favorite singer was also asked from the patient to know their preference and also the type of music such as rock, pop, disco, classical or religious.

\section{Dependent Variable}

1. Post procedure anxiety measured through the dentist rating of patient's scale

The total score this scale offers is $1-6$, in which

$1-2=$ high

3-4 = medium

$5-6=$ low

Patients responses were marked accordingly based upon the scores.

\section{Covariates}

1. Age: Age was asked from the patient and it was divided into categories at the time of analysis such as 18-30 years, 31-45 years and older than 45 years.

2. Gender: both male and female were included in the study.

3. History of presenting complains: history was asked and presenting complain was noted. Responses were recorded as mentioned and at the time of analysis it was post coded into Pain, Hot and cold sensation and Dental carries.

4. Pre procedure anxiety: it was assessed using the Dentist rating of patient's scale.

The total score this scale offers is 1-6, in which $1-2=$ high 
3-4 = medium

$5-6=$ low

Patients responses were marked accordingly based upon the scores

\section{Methods for Data Collection}

A Performa was developed and it consisted of:

1. Bio data part: This part included name of the participant, gender and age of the participant along with his or her occupation and history of presenting complain. Along with it, address and contact number of the patients were also recorded.

2. Dentist rating of patient's scale: anxiety scores of patients in both groups were recorded as pre procedure anxiety and post procedure anxiety.

\section{Data Collection Tools}

Data was collected using the Performa and the dentist rating scale: tools were formulated in English and then after correction it was translated in Urdu and Sindhi as well.

\section{Data Management}

Data collection was carried out by principal investigator of the study. The data collection was carried out in one same day for pre and post procedure anxiety assessment. The completed forms were maintained throughout by the principal investigator and were kept under a lock and key. An electronic database was also maintained by the investigator and data was secured as a soft copy in a hard dick protected by password. Data was entered in the database formed on SPSS. Data entry was also done by the principal investigator.

\section{Ethical Considerations}

All underlying principles of ethics in healthcare research have been taken into consideration from inception till implementation of the study and also after completion of study. In the clinic, proper informed consent was taken and all possible pros and cons were informed to the patient.

\section{Statistical Analysis}

Descriptive statistics were computed. Frequency and percentage has been reported for the categorical variable such as gender, history of presenting complain and occupation while. Mean and standard deviation (SD) has been reported for the quantitative variable such as age. Basic demographic characteristics have been seen with respect to the intervention and control groups as well as overall numbers are also reported.

After computing descriptive statistics, Simple Linear Regression was performed at the Univariate level to assess individual relationship of each independent variable with the outcome variable. At Univariate level variables with $p$-value of $<0.25$ were considered eligible for multivariable analysis.

\section{RESULTS}

Descriptive statistics were computed. Table-I provides the basic demographic characteristics of the participants divided into the intervention group and the control group. Hundred participants were randomized in each group. Out of 200 patients, more than half of the patient belonged to the age group 31 to 45 years. Similarly $62 \%$ of the patients in intervention group belonged to the age group 31 to 45 years and $58 \%$ of the patients in the control group. Table-I tells the pre and post procedure of anxiety level among participants. Table-Il tells the basic demographic variables and their pre and post procedure anxiety score.

Univariate analysis was done to identify the association between the music distraction and the post anxiety level of the participants TableIII. Music intervention was found significant with p-value 0.003 and beta coefficient -0.17 indicating the positive association of music with decrease in anxiety of the patients post procedure. In addition, age gender and history of presenting complain were also entered into the univariate analysis but were found insignificant. Occupation was also entered and business was found to be associated with the post anxiety score i.e. person being involved in business tend to score lower on post anxiety score. Moreover, the preanxiety score was also found insignificant at the univariate level.

For Multivariable analysis, only two variables i.e. 
Music and the occupation of the patient were found significant at the Univariate level and hence they were entered in to the regression. At the multivariable level only music was found significant Table-IV.

\section{DISCUSSION}

This study found an association between the use of music distraction technique and post anxiety score of the participants indicating music distraction as a useful management technique for treating or reducing dental anxiety.

This study also emphasize on the effectiveness of music as a management technique for dental anxiety. A study also established to see the effectiveness of music among patients with dental anxiety found that in the music group the anxiety level was shown to be reduced, confirming the physiologic relaxation due to music distraction. ${ }^{17}$ This finding is consistent with the finding of our study reported.

\begin{tabular}{|c|c|c|c|}
\hline Variables & $\begin{array}{c}\text { Intervention Group }(n=100) \\
n \%\end{array}$ & $\begin{array}{c}\text { Control Group }(n=100) \\
n \%\end{array}$ & $\begin{array}{c}\text { Overall }(n=200) \\
n(\%)\end{array}$ \\
\hline $\begin{array}{l}\text { Age } \\
18 \text { to } 30 \\
31 \text { to } 45 \\
>45\end{array}$ & $\begin{array}{c}36(36) \\
62(62) \\
2(2)\end{array}$ & $\begin{array}{l}28(28) \\
58(58) \\
14(14)\end{array}$ & $\begin{array}{c}64(32) \\
120(60) \\
16(8)\end{array}$ \\
\hline Age mean (SD) & $33.43(7.19)$ & 35.54 (8.29) & $34.49(7.81)$ \\
\hline Variable & Pre-procedure & \multicolumn{2}{|c|}{ Post procedure } \\
\hline $\begin{array}{l}\text { Anxiety Level } \\
\text { Low } \\
\text { Medium } \\
\text { High }\end{array}$ & $\begin{array}{c}0 \\
193(96.5) \\
7(5.5)\end{array}$ & \multicolumn{2}{|c|}{$\begin{array}{c}43(21.5) \\
157(78.5) \\
0\end{array}$} \\
\hline $\begin{array}{l}\text { Anxiety Score } \\
\text { mean (SD) }\end{array}$ & $3.33 \quad(0.611)$ & \multicolumn{2}{|c|}{$4.0 \quad(0.81)$} \\
\hline
\end{tabular}

Table-I. Distribution of age among intervention and control group, pre \& Post procedure anxiety level

\begin{tabular}{|c|c|c|}
\hline Variables & $\begin{array}{l}\text { Anxiety Score } \\
\text { Pre-procedure }\end{array}$ & $\begin{array}{l}\text { Anxiety score } \\
\text { Post-procedure }\end{array}$ \\
\hline $\begin{array}{l}\text { Age } \\
18 \text { to } 30 \\
31 \text { to } 45 \\
>45\end{array}$ & $\begin{array}{ll}3.31 & (0.66) \\
3.33 & (0.60) \\
3.38 & (0.5)\end{array}$ & $\begin{array}{c}3.97(0.84) \\
3.94(0.81) \\
4.0(0.73)\end{array}$ \\
\hline $\begin{array}{l}\text { Gender } \\
\text { Male } \\
\text { Female }\end{array}$ & $\begin{array}{c}3.23(0.50) \\
3.40(0.67)\end{array}$ & $\begin{array}{l}3.99(0.80) \\
3.93(0.82)\end{array}$ \\
\hline $\begin{array}{l}\text { Occupation } \\
\text { no occupation } \\
\text { student } \\
\text { business } \\
\text { job/service } \\
\text { manual laborer } \\
\text { housewife }\end{array}$ & $\begin{array}{c}3.45(0.76) \\
3.28(0.72) \\
3.12(0.33) \\
3.22(0.55) \\
3.44(0.56) \\
3.39(0.59)\end{array}$ & $\begin{array}{c}3.95(0.51) \\
4.0(0.91) \\
4.29(1.11) \\
3.91(0.73) \\
3.97(0.78) \\
3.85(0.78)\end{array}$ \\
\hline $\begin{array}{l}\text { History of Presenting Complains } \\
\text { Pain } \\
\text { Hot and cold sensation } \\
\text { Dental carries }\end{array}$ & $\begin{array}{l}3.36(0.59) \\
3.32(0.65) \\
3.10(0.54)\end{array}$ & $\begin{array}{ll}3.98 & (0.79) \\
3.94 & (0.84) \\
3.73 & (0.91)\end{array}$ \\
\hline $\begin{array}{l}\text { Music } \\
\text { Given } \\
\text { Not Given }\end{array}$ & $\begin{array}{cc}3.21 & (0.69) \\
3.45 & (0.5)\end{array}$ & $\begin{array}{ll}4.11 & (0.92) \\
3.8 & (0.65)\end{array}$ \\
\hline
\end{tabular}

Table-II. Basic demographic variables and their pre and post procedure anxiety scores 


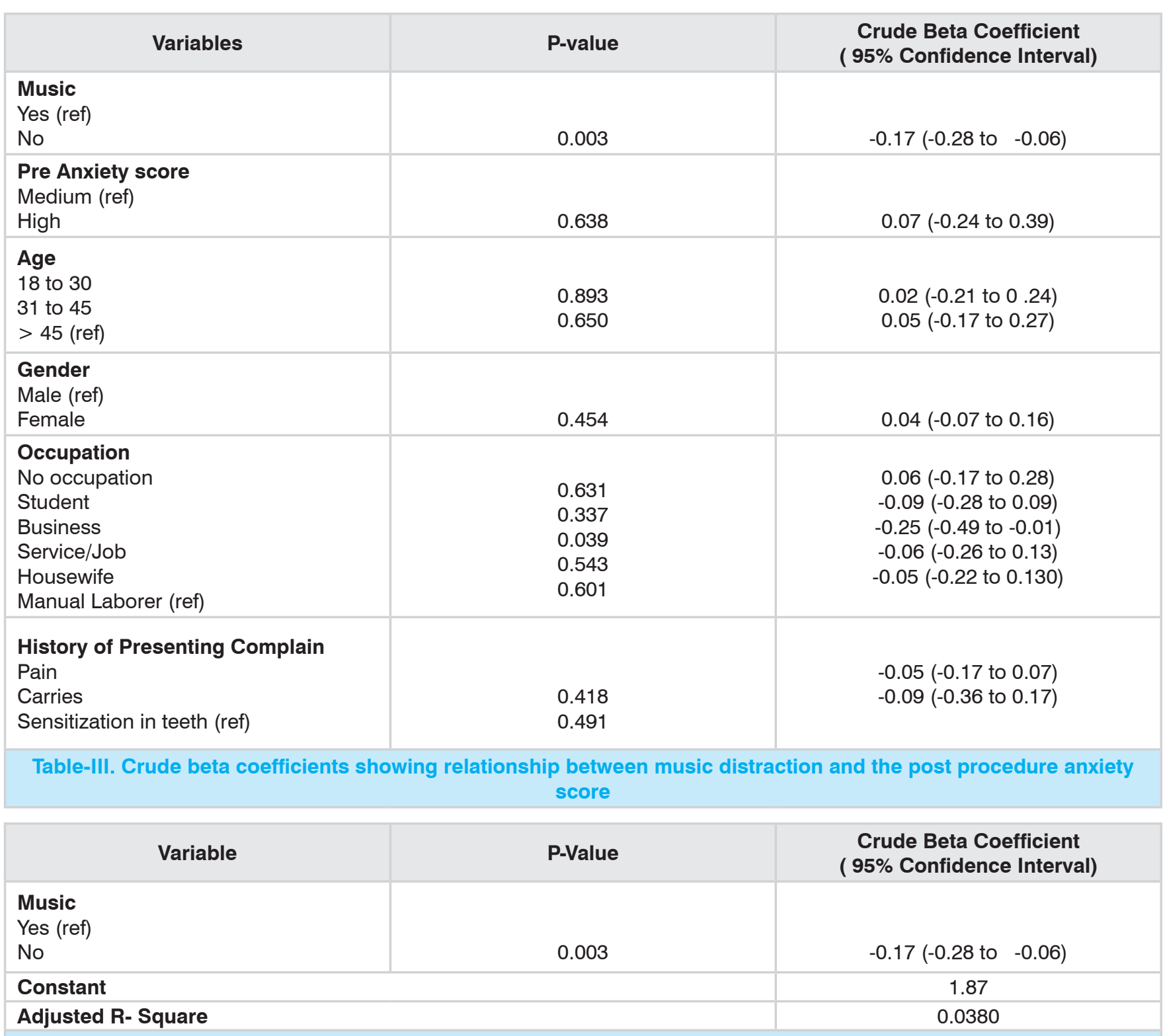

Table-IV. Final model showing association between music and post anxiety score

Literature also reports various factors that are associated with dental anxiety of them age and gender are the mostly studied variables in conjunction with the dental anxiety. Dental anxiety has been shown to vary according to age and sex. ${ }^{18}$ Studies done to identify factors associated with dental anxiety revealed that anxiety decreases with age while Appukutian's found no association of age with dental anxiety in his study "Prevalence of dental anxiety among patients attending a dental educational institution in Chennai". ${ }^{18}$ Studies have also shown that "with age fears and phobias are decline, which could be attributed to age dependent cerebral deterioration, factors like extinction or habituation, and adaptive resignation towards the inevitable". ${ }^{18}$ In our study Age group 18-30 years, 31 to 45 years and 45 above were analyzed for its association with the post procedure dental anxiety score and it has been found that there is no association of age with the score patient marked on the post procedure anxiety scale.

In addition to age, gender is also considered a predictor for dental anxiety. Literature highlight that there is a gender difference in the experience 
of dental anxiety. Females are more inclined to experience dental anxiety compared to man, ${ }^{19}$ and however, one of the study of adults aged 50 years and older found no difference in dental anxiety according to sex. ${ }^{18}$ Similarly our study didn't build upon the findings according the literature reports. In this study there is no association between the gender and the post anxiety score of the participants.

According to a study conducted in India among Tamil speaking population it was found that there was no gender differences in the anxiety level which is consistent with the finding that our study discovered. Causes of dental anxiety when inquired among them revealed that "tooth drilling and local anesthetic injection, were the most common reasons for anxiety. That study also found that younger respondents, uneducated, unemployed and lower income group were dentally more anxious and also showed that dentally anxious patients postponed their dental visit and past negative dental experiences were associated with significant anxiety scores". ${ }^{18}$

Our study did not find any predictive factor associated with dental anxiety. There could be several reasons for that, first of all the studies which are being looked upon for the association of music and dental anxiety and factors of dental anxiety were carried out among different population i.e. there geography differs and their health seeking behavior also differs when compared to Pakistani population. There could be possibility that the factors which other studies found significant are not considered important predictors of dental anxiety in our population.

\section{CONCLUSION}

Dental anxiety is a frequently encountered condition among patients worldwide. Many management techniques have been used till date. Few of them are still in use based upon their effectiveness while others are considered redundant due to their cost, Side effects or time consuming nature. Music is a simple and time saving intervention. Dental professionals who use music in the routine dental care procedures are more likely to develop rapport with the patients.
Likewise patient would appreciate the efforts done by their physicians in helping them dealing with the threatening situations.

Copyright (C) 15 Dec, 2018.

\section{REFERENCES}

1. Joanna Briggs I. Music interventions for dental anxiety in paediatric and adult patients. The JBI Database of Best Practice Information Sheets and Technical Reports. 2011.15(2):1-4.

2. Mok E, Wong KY. Effects of music on patient anxiety. AORN J 2003; 77(2):396-7, 401-6, 409-10.

3. Economou GC. Dental anxiety and personality: Investigating the relationship between dental anxiety and self-consciousness. J Dent Educ. 2003 Sep; (9):970-80.

4. Wakim JH, Smith S, Guinn C. The efficacy of music therapy. Journal of perianesthesia nursing. 2010. 25(4):226-32 .

5. Bare LC, Dundes L. Strategies for combating dental anxiety. J Dent Educ. 2004; 68(11):1172-7.

6. Freeman R.E. Dental Anxiety: A multifactorial aetiology. Br Dent J 1985; 159(12):406-8.

7. Armfield JM, Heaton LJ. Management of fear and anxiety in the dental clinic: A review. Australian dental journal.2013. 58(4):390-407.

8. Ogle $\mathrm{OE}$, Hertz MB. Anxiety control in the dental patient. Dental Clinics of North America. 2012. 56(1):116.

9. Gatchel RJ, Ingersoll BD, Bowman L, et al. The prevalence of dental fear and avoidance: $A$ recent survey study. J Am Dent Assoc 1983; 107(4):609-10.

10. DeNitto VM. Efficacy of audiovisual distraction in the reduction of dental anxiety during endodontic treatment. 2012.

11. Chanpong B, Haas DA, Locker D. Need and demand for sedation or general anesthesia in dentistry: A national survey of the Canadian population. Anesth Prog 2005; 52(1):3-11.

12. Chiu W-s. Effect of music on anxiety management during dental procedures: The University of Hong Kong (Pokfulam, Hong Kong) 2010.

13. Wakim JH, Smith $\mathrm{S}$, Guinn $\mathrm{C}$. The efficacy of music therapy. J Peri Anesth Nurs 2010; 25(4):226-32.

14. Klassen JA, Liang Y, Tjosvold L, Klassen TP, Hartling L. Music for pain and anxiety in children undergoing 
medical procedures: A systemic review of randomized controlled trails. Ambul Pediatr 2008; $8(2): 117-28$.

15. Berggren U, Meynert G. Dental fear and avoidance: causes, symptoms and consequences. J Am Dent Assoc 1984; 109(2):247-51.

16. Olszewska I, Zarow M. Does Music during a dental treatment make a difference. J Dent Res. 2003; 82.

17. Sandeep N, Bailwad A, Nirmala S, Sivakumar N. Effectiveness of music distraction in the management of anxious pediatric dental patients. Annals and essences of dentistry. 2010; 2(2):1-5.

18. Appukuttan DP, Tadepalli A, Cholan PK. Prevalence of dental anxiety among patients attending a dental educational institution in Chennai, India " $A$ questionnaire based study. Oral health and dental management. 2013.

19. Wide Boman U, Carlsson V, Westin M, Hakeberg M. Psychological treatment of dental anxiety among adults: A systematic review. European journal of oral sciences.2013. 121(3pt2):225-34.

\title{
Forgiveness says you are given another chance to make a new beginning.
}

\author{
"Desmond Tutu"
}

\begin{tabular}{|c|l|l|l|}
\hline \multicolumn{3}{|c|}{ AUTHORSHIP AND CONTRIBUTION DECLARATION } \\
\hline Sr. \# & \multicolumn{1}{|c|}{ Author-s Full Name } & \multicolumn{1}{|c|}{ Contribution to the paper } & Author=s Signature \\
\hline 1 & Abdul Qadir Khan & Concept, Result, Discussion. \\
\hline 2 & Batool Bibi & Data collection. \\
\hline 3 & $\begin{array}{l}\text { Ahmed Babikar } \\
\text { Mohamed Ali } \\
\text { Saifullah Qureshi }\end{array}$ & $\begin{array}{l}\text { Introduction, Conclusion, } \\
\text { References. } \\
\text { Critical analysis, article searching. }\end{array}$ \\
\hline
\end{tabular}

